\title{
Introducing physical relaxation terms in Bloch equations
}

\author{
B. Bidégaray ${ }^{\dagger}$, A. Bourgeade ${ }^{\ddagger}$ and D. Reignier ${ }^{\dagger 1}$ \\ ${ }^{\dagger}$ Laboratoire MIP - CMRS UMR 5640, 118 route de Narbonne, F-31062 Toulouse Cedex 4 , \\ ${ }^{\ddagger}$ CEA-CESTA, BP 2, F-33114 Le Barp \\ E-mail: bidegara@mip.ups-tlse.fr; bourgead@bordeaux.cea.fr; didier.reignier@fnac.net
}

Bloch equation models the evolution of the state of electrons in a matter described by an Hamiltonian. In order to model more physical phenomena we have to introduce phenomenological relaxation terms. The introduction of these terms has to conserve some positiveness properties. The aim of this paper is to give a review of possible relaxation models as well as an insight of how to discretize them properly in view of numerical computations.

Key Words: Bloch equations, relaxation terms, quantum optics

\section{INTRODUCTION}

Nowadays laser sources allow to produce light pulses that are increasingly powerful and shorter. The propagation of such beams through a medium induces nonlinear light-matter interactions. Moreover as the pulse duration may be of the same order or even much smaller than the time response of the medium, transient phenomena have to be considered. Thus models will take place in the time-domain.

The classical description of the propagation of an electromagnetic wave will be done by Maxwell equations, the influence of the matter being expressed through the polarization. A simple description involving the refractive index is not sufficient, even when frequency and intensity dependent. Here a very precise modeling is required for matter. This is performed by Bloch equations which are derived in the context of quantum mechanics. These equations deal with the probabilistic description of the population of each energy level of atoms that constitutes the matter as well as the coherence between these levels. These variables are gathered in a so-called density matrix. The statistical averaging over atoms is taken into account by the introduction of relaxation terms. These terms are also necessary to model some important physical phenomena as spontaneous emission, collisions,

${ }^{1}$ This work has been partially supported by a grant from the CEA-CESTA, No 8V2011YBF3 
etc. There are different classical ways to introduce them $[3,4,6]$ but we present here "Pauli's master equation" model which happens to be the more general one.

The relaxation terms that we introduce should preserve some important properties that would be valid without these additional terms. First populations have to be positive and less than 1 . Coherences should not be greater than the related populations and density matrices should be positive matrices. As will be stressed below, in the absence of statistical averaging (and therefore relaxation) all these properties are straightforward.

We do not know of any existing literature about this specific problem of conservation of positiveness properties when adding relaxation terms. The reasons may be the following. As we show, positiveness is conserved in all classical physical contexts, hence this is not a problem for physicists. From the numerical point of view, Bloch codes were up to now involving only two-level atoms [5, 8, 10] and problems occur with at least three levels. Such a study is useful for our multi-level code and the simulation of physical contexts that involve more than two levels and different relaxation terms, like a laser cavity for example. The scope of this article is limited to the Bloch equations with a given electromagnetic field, the problem of the coupling with a propagation model being postponed for further articles.

In section 2 we give a brief description of Bloch equations. Section 3 is devoted to the introduction of "Pauli's master equation" model. A time semi-discretization of Bloch equation is dealt with in section 4 . This discretization conserves positiveness and is tractable for real numerical computations.

\section{BLOCH EQUATIONS}

In quantum mechanics, matter is defined by state vectors $|\psi\rangle$, the time evolution of which is given by the Schrödinger equation

$$
i \hbar \partial_{t}|\psi\rangle=H|\psi\rangle
$$

The Hamiltonian $H$ is composed of the unperturbed Hamiltonian $H_{0}$ and the perturbation $V$ induced by the electromagnetic field : $H=H_{0}+V$. Following a standard approach we decompose $|\psi\rangle$ on the basis of eigenstates of the unperturbed Hamiltonian $H_{0}$. These quantum states $|j\rangle$ are the eigenfunctions of $H_{0}$ that correspond to the $j^{\text {th }}$ level of energy $\mathcal{E}_{j}=\hbar \omega_{j}$ of unperturbed atoms. The set of all these (suitably normalized) quantum states forms a basis of all quantum states. We therefore may set $|\psi\rangle=\sum a_{j}|j\rangle$ and the (infinite dimensional) density matrix $\rho$ is defined by $\rho_{j k}=\sum_{S} a_{j}{ }^{S} a_{k}^{* S}$ where $S$ is a statistical set. It is solution to

$$
i \hbar \partial_{t} \rho=[H, \rho]
$$

where $[\cdot, \cdot]$ denotes the commutator of two operators. Keeping only $N$ relevant levels, $\rho$ is a $N \times N$ hermitian non negative matrix. Its diagonal elements $\rho_{j j}$ represent the population of levels $|j\rangle$ and its off-diagonal elements $\rho_{j k}$ the coherences between 
To express the perturbation $V$, we restrict ourselves to dipole moments because they induce the larger order in the perturbation series. Perturbation $V$ reads $V=$ $-e \vec{E} \cdot \vec{R}$ having taken into account the fact that $\vec{E}$ does not significantly vary over atomic distances. The dipole moment matrix is defined by its elements $\vec{p}_{j k}=$ $\langle k|e \vec{R}| j\rangle$. With these notations equation (1) reads

$$
\partial_{t} \rho_{j k}=-i \omega_{j k} \rho_{j k}-\frac{i}{\hbar} \vec{E} \cdot[\vec{p}, \rho]_{j k},
$$

where $\omega_{j k}=\omega_{j}-\omega_{k}$ is the frequency associated to the transition from level $|k\rangle$ to level $|j\rangle$.

In the expression of $V, \vec{E}$ may be a given field or may be the solution of Maxwell equations. In the latter case there is a coupling between Bloch equations and Maxwell equations via the constitutive relation $\vec{D}=\varepsilon_{0} \vec{E}+\vec{P}$ where the polarization $\vec{P}$ is given in terms of the density matrix by $\vec{P}=N_{a} \operatorname{Tr}(\vec{p} \rho)$ and $N_{a}$ is the density of atoms.

\section{3. "PAULI'S MASTER EQUATION" MODEL}

To derive the Bloch equations the following hypothesis is made: the stochastic process for driving the statistical distribution $S$ is stationary. This is however not the case and besides it is not possible to obtain any information about this process. Other phenomena are not included in the original model, the main of which being spontaneous emission of light, but also collisions, vibrations in crystal lattices or thermal perturbations in fluids. The only way to take all these phenomena into account is to add phenomenological relaxation terms to equation (2) that becomes

$$
\partial_{t} \rho_{j k}=-i \omega_{j k} \rho_{j k}-\frac{i}{\hbar}[V, \rho]_{j k}+Q(\rho)_{j k} .
$$

The addition of relaxation terms for a coherence only involves this variable and therefore for $j \neq k, Q(\rho)_{j k}=-\gamma_{j k} \rho_{j k}$. Models for diagonal relaxation terms are more various but may be shown to be sub-models of "Pauli's master equation" model where

$$
Q(\rho)_{j j}=\sum_{l \neq j} W_{j l} \rho_{l l}-\sum_{l \neq j} W_{l j} \rho_{j j}=\sum_{l \neq j} W_{j l} \rho_{l l}-\Gamma_{j} \rho_{j j} .
$$

The relaxation to equilibrium states is obtained by imposing

$$
W_{j l}=W_{l j} e^{\beta\left(\mathcal{E}_{j}-\mathcal{E}_{l}\right)}
$$

(see e.g. Bloembergen [3]). Here $\beta=1 / \kappa T$, where $\kappa$ is Boltzmann's constant and $T$ is the temperature.

In what follows we will always suppose that some physical properties are satisfied for the initial data and try to find conditions on the relaxation operator to propagate them for all time. 


\subsection{Physical properties}

The most obvious property for $\rho$ is that it should be hermitian (i.e. it is an observable quantity from the quantum mechanical point of view). The only way to ensure this property is that equations for $\rho_{j k}$ and $\rho_{k j}$ are conjugate. Therefore $\gamma_{j k}$ and $\gamma_{k j}$ should be equal, which is indeed the choice that is always made.

All relevant energy levels are supposed to be kept in the model which may be expressed as $\operatorname{Tr}(\rho)=1$. This property is conserved since (3) yields a priori $\partial_{t} \operatorname{Tr}(\rho)=\operatorname{Tr}(Q(\rho))$ and the specific relaxation operator $Q$ given by (4) ensures that $\operatorname{Tr}(Q(\rho))=0$.

The other properties we want to preserve while adding relaxation terms are positiveness properties. First of all populations have to be nonnegative. They also have to be less than one but this is a consequence of positiveness and the above trace property. The coherence of two levels has also to be controlled by the population of both levels or more precisely $\left|\rho_{j k}\right|^{2} \leq \rho_{j j} \rho_{k k}$. Finally the whole matrix has to be nonnegative.

\subsection{Positiveness}

In order to study positiveness we note that it is preserved by the relaxation-free model (see section 4.1 below) and we use Trotter-Kato formula that ensures that

$$
\rho(t)=e^{t(L+Q)} \rho(0)=\lim _{n \rightarrow \infty}\left(e^{\frac{t L}{n}} e^{\frac{t Q}{n}} \rho(0)\right)^{n},
$$

where $L \rho_{j k}=-i \omega_{j k} \rho_{j k}-\frac{i}{\hbar}[V, \rho]_{j k}$. Therefore it is sufficient that the equation

$$
\partial_{t} \rho=Q(\rho)
$$

preserves positiveness properties. In the sequel of this section proofs address this equation. Besides all the proofs suppose that the quantities we study are nonnegative at time $t=0$.

Positiveness of populations. Given a level $k$, we consider the initial data $\rho_{j j}=$ $\delta_{j k}$. For $j \neq k$, we have $\partial_{t} \rho_{j j}(0)=W_{j k}$. Since $\rho_{j j}(0)=0$, its derivative should not be negative and therefore a necessary condition is $W_{j k} \geq 0$.

This is also a sufficient condition: if at some time $t_{0}, \rho_{j j}\left(t_{0}\right)=0$ then $\partial_{t} \rho_{j j}\left(t_{0}\right)=$ $\sum_{k \neq j} W_{k j} \rho_{k k}\left(t_{0}\right)$. Since there exists $k_{0} \neq j$ such that $\rho_{k k}\left(t_{0}\right) \neq 0$ (see the trace property), we show that if $W_{j k_{0}}>0$ either $t_{0}=0$ and for $t>0$, but small, $\rho_{j j}(t)>0$ either $t_{0} \neq 0$ and it is not possible that $\rho_{j j}\left(t_{0}\right)=0$. Thus if $W$ is a matrix with positive coefficients, $\rho_{j j}(t)$ is positive for $t>0$. Besides $\rho(t)$ is continuous with respect to the matrix $W$, hence if $W$ is only nonnegative $\rho_{j j}(t)$ remains nonnegative for all time $t>0$. The first condition on matrix $W$ is the following.

Condition 3.1. A necessary and sufficient condition for populations to be nonnegative (and less than 1 via the trace property) is that matrix $W$ has nonnegative coefficients. 
We notice that this first condition is always verified from a physical point of view.

Estimate for the coherences. We set $f(t) \equiv \rho_{j j}(t) \rho_{k k}(t)-\rho_{j k}(t) \rho_{k j}(t)$ and suppose that $f\left(t_{0}\right)$ is zero for some time $t_{0}$ but are not interested in the case when $\rho_{j j}\left(t_{0}\right) \rho_{k k}\left(t_{0}\right)=0$, i.e. $\rho_{j k}\left(t_{0}\right)=0$, for which $\rho_{j k} \equiv 0$ for all time. We notice that $\rho_{j j}(t) \rho_{k k}(t) \geq 0$ is the consequence of condition 3.1. Computing $f^{\prime}$ we obtain for a general time $t$

$$
\begin{aligned}
f^{\prime}(t) & =2 \gamma_{j k} f(t)+\left(2 \gamma_{j k}-\Gamma_{j}-\Gamma_{k}+\sqrt{W_{j k} W_{k j}}\right) \rho_{j j}(t) \rho_{k k}(t) \\
& +\left(W_{j k} \rho_{k k}-W_{k j} \rho_{j j}\right)^{2}+\rho_{j j}(t) \sum_{l \neq j, k} W_{k l} \rho_{l l}(t)+\rho_{k k}(t) \sum_{l \neq j, k} W_{j l} \rho_{l l}(t)
\end{aligned}
$$

If $2 \gamma_{j k}-\Gamma_{j}-\Gamma_{k}+\sqrt{W_{j k} W_{k j}}>0$ we show (in the same way than above) that $f(t)$ is positive for all positive time. A continuity argument gives the following condition.

CONDITION 3.2. A necessary and sufficient condition to have the estimate

$$
\left|\rho_{j k}(t)\right| \leq \sqrt{\rho_{j j}(t) \rho_{k k}(t)}
$$

for all time $t \geq 0$ is that $2 \gamma_{j k} \geq \Gamma_{j}+\Gamma_{k}-\sqrt{W_{j k} W_{k j}}$.

The fact that the condition is necessary is proved supposing that $\rho_{l l}=0$ for $l \neq$ $j, k$. Condition 3.2 is also a physical condition (see e.g. [7]). Off-diagonal decay rates have the same source than diagonal decay rates plus some extra sources like elastic collision broadening, ... Therefore $\gamma_{j k}$ is often written as $\gamma_{j k}=\frac{1}{2}\left(\Gamma_{j}+\Gamma_{k}\right)+\gamma_{j k}^{\text {coll }}$, where $\gamma_{j k}^{\text {coll }} \geq 0$. In many physical contexts we even have $\gamma_{j k}^{\text {coll }} \gg \Gamma_{j}+\Gamma_{k}$. Moreover $\gamma_{j k}^{c o l l}$ very often does not depend on $j$ and $k$.

For the sequel we are unable to treat a condition including the expression $\sqrt{W_{j k} W_{k j}}$, but we give a specific form for $\gamma_{j k}$ that covers all physical models:

$$
\gamma_{j k}=\frac{1}{2}\left(\Gamma_{j}+\Gamma_{k}\right)+\gamma_{j}^{c o l l}+\gamma_{k}^{\text {coll }}-A_{j} \cdot A_{k}
$$

where $\gamma_{j}^{\text {coll }} \in \mathbb{R}$ and $A_{j} \in \mathbb{R}^{N}$.

Positiveness of the density matrix. Let $X=\left(x_{1}, \ldots, x_{N}\right) \in \mathbb{C}^{N}$ and $g(t)=$ $X^{*} \rho(t) X$. We suppose that $t_{0}$ is the first time for which $g\left(t_{0}\right)=0$. For this time $\rho\left(t_{0}\right)$ is a hermitian nonnegative matrix and we may state that $\rho\left(t_{0}\right) X=0$ and $X^{*} \rho\left(t_{0}\right)=0$.

$$
\begin{aligned}
g^{\prime}(t) & =\sum_{j} \sum_{k \neq j} W_{j k} \rho_{k k}\left|x_{j}\right|^{2}-\sum_{j} \Gamma_{j} \rho_{j j}\left|x_{j}\right|^{2}-\sum_{j, k, j \neq k} \gamma_{j k} x_{j}^{*} \rho_{j k} x_{k} \\
& =\sum_{j} \sum_{k \neq j} W_{j k} \rho_{k k}\left|x_{j}\right|^{2}+\sum_{j}\left(2 \gamma_{j}^{c o l l}-\left\|A_{j}\right\|^{2}\right) \rho_{j j}\left|x_{j}\right|^{2}
\end{aligned}
$$




$$
\begin{aligned}
& \quad-\sum_{j}\left(\frac{1}{2} \Gamma_{j}+\gamma_{j}^{\text {coll }}\right) x_{j}^{*}(\underbrace{\left.\sum_{k} \rho_{j k} x_{k}\right)}_{=0}-\sum_{k}\left(\frac{1}{2} \Gamma_{k}+\gamma_{k}^{\text {coll }}\right) \underbrace{\left(\sum_{j} x_{j}^{*} \rho_{j k}\right)}_{=0} x_{k} \\
& +\sum_{k} \underbrace{\sum_{j} \sum_{k} \gamma_{k}\left(a_{j}^{l} x_{j}\right)^{*} \rho_{j k}\left(a_{k}^{l} x_{k}\right)}_{\geq 0} \\
& =\sum_{j} \sum_{k \neq j} W_{j k} \rho_{k k}\left|x_{j}\right|^{2}+\sum_{j}\left(2 \gamma_{j}^{\text {coll }}-\left\|A_{j}\right\|^{2}\right) \rho_{j j}\left|x_{j}\right|^{2} \\
& \quad+\sum_{l} \underbrace{\sum_{j} \sum_{k} \gamma_{k}\left(a_{j}^{l} x_{j}\right)^{*} \rho_{j k}\left(a_{k}^{l} x_{k}\right)}_{\geq 0} .
\end{aligned}
$$

A sufficient condition for $g(t)$ to be positive is therefore $\gamma_{j}^{\text {coll }}>\frac{1}{2}\left\|A_{j}\right\|^{2}$ for all $j$ and it is relaxed by continuity to the condition for $g(t)$ to be nonnegative $\gamma_{j}^{\text {coll }} \geq \frac{1}{2}\left\|A_{j}\right\|^{2}$.

CONDITION 3.3. With the hypothesis (7) on collisional decay rates, a sufficient condition for $\rho$ to be a nonnegative matrix for all time is $\gamma_{j}^{\text {coll }} \geq \frac{1}{2}\left\|A_{j}\right\|^{2}$ for all $j$.

We note that condition 3.3 is more general than 3.2. Besides the most widely used physical model corresponds to $A_{j} \equiv 0$ and $\gamma_{j}^{\text {coll }}=\frac{1}{2} \gamma^{\text {coll }}$, i.e. $\gamma_{j k}=\frac{1}{2}\left(\Gamma_{j}+\right.$ $\left.\Gamma_{k}\right)+\gamma^{\text {coll }}$.

Remark. Condition 3 may be written $2 \gamma_{j k}=\Gamma_{j}+\Gamma_{k}+\left\|B_{j}-B_{k}\right\|$ for $B_{j} \in \mathbb{C}^{N}$.

\section{NUMERICAL ISSUES}

The most commonly used time discretization for the Bloch equations is the Crank-Nicolson scheme. For example Ziolkowski, Arnold and Gogny [10], who study the Maxwell-Bloch equation, use this scheme coupled to a Yee scheme for the Maxwell equation. Nagra and York [9], when studying a coupling between Maxwell equations and rate equation (involving only populations), use also this centered scheme. We show that this approach is indeed valid when dealing with two-level Bloch equations. But for a greater number of energy levels positiveness is no longer preserved and for example negative populations may be actually observed numerically.

Some other methods are also present in the literature. Martín et al. [8] use a multigrid approach with a leap-frog scheme in the case of two level atoms and in the slowly varying envelope approximation for the electromagnetic field. There would be at least two ways to write their scheme in our context, since for two level equations the coherences do not appear in the expression of the interaction terms in the evolution equation for the coherences. This scheme is also not positiveness preserving.

We give an alternative discretization for the Bloch equation that do apply in a general framework. We do not comment on how the Bloch equations may be coupled to a model for the propagation of the electromagnetic field. Indeed this problem which is important for full Maxwell-Bloch simulations is not directly connected 
to the introduction of relaxation terms and to the conservation of positiveness properties. We refer to [1] for a discussion on the time coupling of Maxwell and Bloch equations.

\subsection{Analysis of the Crank-Nicolson scheme}

The problem for the Crank-Nicolson is not connected with the relaxation operator therefore we explain it on the relaxation free model (1). Given a time step $\delta t$, the Crank-Nicolson scheme reads

$$
\frac{\rho^{n+1}-\rho^{n}}{\delta t}=-\frac{i}{\hbar}\left(H^{n+1 / 2} \frac{\rho^{n+1}+\rho^{n}}{2}-\frac{\rho^{n+1}+\rho^{n}}{2} H^{n+1 / 2}\right)
$$

where $\rho^{n}$ and $H^{n+1 / 2}$ are respectively approximations of $\rho(n \delta t)$ and $H\left(n \delta t+\frac{\delta t}{2}\right)$. We choose approximations such that $H^{n+1 / 2}$ is hermitian and therefore we may diagonalize it in some basis yielding $\tilde{H}^{n+1 / 2}=\operatorname{diag}\left(\lambda_{1}^{n+1 / 2}, \ldots, \lambda_{N}^{n+1 / 2}\right)$. Writing $\rho^{n}$ in this basis gives $\tilde{\rho}^{n}$ that is solution to

$$
\frac{\tilde{\rho}_{j k}^{n+1}-\tilde{\rho}_{j k}^{n}}{\delta t}=-\frac{i}{\hbar}\left(\lambda_{j}^{n+1 / 2} \frac{\rho_{j k}^{n+1}+\rho_{j k}^{n}}{2}-\lambda_{k}^{n+1 / 2} \frac{\rho_{j k}^{n+1}+\rho_{j k}^{n}}{2}\right)
$$

or explicitly

$$
\tilde{\rho}_{j k}^{n+1}=\frac{1-\frac{i \delta t}{2 \hbar}\left(\lambda_{j}^{n+1 / 2}-\lambda_{k}^{n+1 / 2}\right)}{1+\frac{i \delta t}{2 \hbar}\left(\lambda_{j}^{n+1 / 2}-\lambda_{k}^{n+1 / 2}\right)} \tilde{\rho}_{j k}^{n} .
$$

This does not lead to a positive matrix $\tilde{\rho}^{n+1}$ provided $\tilde{\rho}^{n}$ is positive. To understand this point we have to compare with the continuous case. We may diagonalize the system (1) for all time $t$ and obtain

$$
i \hbar \tilde{\rho}_{j k}^{\prime}(t)=\left(\lambda_{j}(t)-\lambda_{k}(t)\right) \tilde{\rho}_{j k}(t)
$$

and for all $X=\left(x_{1}, \ldots, x_{N}\right) \in \mathbb{C}^{N}, \sum_{j k} x_{j}^{*} \tilde{\rho}_{j k}(t) x_{k}=\sum_{j k} y_{j}^{*} \tilde{\rho}_{j k}(0) y_{k}$ where $y_{j}=x_{j} \exp \left(-\frac{i}{\hbar} \int_{0}^{t} \lambda_{j}(s) d s\right)$. The discrete multiplicator is an approximation of $\exp \left(-\frac{i}{\hbar} \int_{0}^{t}\left(\lambda_{j}(s)-\lambda_{k}(s)\right) d s\right)$ but does not have the property to split in a $j$ and a $k$ contribution. In [10] they only treat two-level atoms and this problem does not occur since there is only one off-diagonal term.

\subsection{An alternative method}

The alternative method we introduce is based on a splitting procedure, i.e. we solve separately

$$
\partial_{t} \rho=L \rho,
$$

where $(L \rho)_{j k}=-i \omega_{j k} \rho_{j k}+Q(\rho)_{j k}$ and

$$
i \hbar \partial_{t} \rho=[V, \rho] .
$$

In equation (8) the linear operator is constant and $\rho(t+\delta t)=\exp (L \delta t) \rho(t)$. Therefore we may compute $\exp (L \delta t)$ once and for all and apply it at each time step. 
Equation (9) is solved exactly by $\rho(t+\delta t)=\exp \left(\frac{i V}{\hbar} \delta t\right) \rho(t) \exp \left(-\frac{i V}{\hbar} \delta t\right)$, but it would be too expensive to implement the diagonalization that is used for the proof above at each time step since $V$ depends a priori on time (and space if the system is coupled to a model for the field). We replace the exact solution by the approximate solution $\rho(t+\delta t) \sim\left(I-\frac{i \delta t}{2 \hbar} V\right)^{-1}\left(I+\frac{i \delta t}{2 \hbar} V\right) \rho(t)\left(I-\frac{i \delta t}{2 \hbar} V\right)\left(I+\frac{i \delta t}{2 \hbar} V\right)^{-1}$ where $V$ is linear with respect to $E$ and the inverse matrices may be computed via Fadeev formula. This leads to a very efficient implementation of the scheme. The advantage of this splitting is that it preserves positiveness for each of these equations. Indeed each step (8) and (9) preserves positiveness. The numerical solution of the first step is exact since we compute matrix $\exp (L \delta t)$ and the approximation of the second step preserves positiveness: $X^{*} \rho(t+\delta t) X=Y^{*} \rho(t) Y$ with $Y=\left(I-\frac{i \delta t}{2 \hbar} V\right)\left(I+\frac{i \delta t}{2 \hbar} V\right)^{-1} X$. Last, with these two steps it is possible to design methods with any order of accuracy, although it is not worthwhile to do so if the model for the electromagnetic field that might be coupled with it has a low order of accuracy.

If we write, for example the second order scheme, we have

$$
\rho^{n+1}=e^{L \delta t / 2}\left(I-\frac{i \delta t}{2 \hbar} V\right)^{-1}\left(I+\frac{i \delta t}{2 \hbar} V\right) e^{L \delta t / 2} \rho^{n}(t)\left(I-\frac{i \delta t}{2 \hbar} V\right)\left(I+\frac{i \delta t}{2 \hbar} V\right)^{-1} .
$$

The appendix shows that $M_{N}=\left(I-\frac{i \delta t}{2 \hbar} V\right)\left(I+\frac{i \delta t}{2 \hbar} V\right)^{-1}$ reads $I+\delta t \phi(V, \delta t)$ where $\phi(V, \delta t)$ is bounded for $\delta t \leq \delta t_{0}$ for any $\delta t_{0}$. The operator $e^{L \delta t / 2}$ also reads $I+$ $\delta t \psi(\delta t)$ where $\psi(\delta t)$ is bounded for $\delta t \leq \delta t_{0}$. Let us gather all the real variables (the diagonal elements, the real and imaginary parts of the off-diagonal elements) of the density matrix $\rho^{n}$ in a vector $y^{n}$. Therefore, we may write the splitting scheme as $y^{n+1}=y^{n}+\delta t \Phi\left(t_{n}, y^{n}, \delta t\right)$ where for all $\delta t_{0}, \Phi\left(t_{n}, y^{n}, \delta t\right)$ is Lipschitz in the second variable uniformly with respect to $\delta t \leq \delta t_{0}$. The dependence in $t_{n}=n \delta t$ comes from $V$ which is supposed to be Lipschitz in time as well (it is a sine function in our test cases). This is a sufficient condition for stability.

\subsection{Some applications}

We present here some computations for Bloch equations with a given electric field. We focus our interest on performances and the illustration of the use of different relaxation terms. Coupling with a Maxwell solver leads to richer physical phenomena and we refer to [2] for examples in a one-dimensional space. Research about higher dimensions is in progress.

\section{Comparison of different numerical schemes.}

In this paragraph we compare three schemes. The first one is the Crank-Nicolson scheme which is also the scheme which is the most widely used. We also tried a relaxation scheme where diagonal terms $\left(\rho_{d}\right)$ are computed at times $n \delta t$ and offdiagonal terms $\left(\rho_{o d}\right)$ at time $\left(n+\frac{1}{2}\right) \delta t$, namely

$$
\begin{aligned}
\frac{\rho_{o d}^{n+1 / 2}-\rho_{o d}^{n-1 / 2}}{\delta t} & =\operatorname{Rn}\left(\frac{\rho_{o d}^{n+1 / 2}+\rho_{o d}^{n-1 / 2}}{2}\right)-\frac{i}{\hbar}\left[V^{n}, \frac{\rho_{o d}^{n+1 / 2}+\rho_{o d}^{n-1 / 2}}{2}+\rho_{d}^{n}\right], \\
\frac{\rho_{d}^{n+1}-\rho_{d}^{n}}{\delta t} & =\operatorname{Rn}\left(\frac{\rho_{d}^{n+1}+\rho_{d}^{n}}{2}\right)-\frac{i}{\hbar}\left[V^{n+1 / 2}, \rho_{o d}^{n+1 / 2}\right] .
\end{aligned}
$$


Such a scheme is of no interest in the case of a forced electromagnetic field but leads to less coupled equations in the case of Maxwell-Bloch equations (see [1]). Last we tested the splitting scheme that we describe in section 4.2. In a twolevel Bloch code one diagonal variable is usually not computed and replaced by the trace conservation law. This would lead to unnecessary complications in the implementation of a multi-level code and therefore we test the different methods with respect to trace conservation, positiveness conservation and CPU times. To show the differences we have to run a large number of iterations, figure 1 shows iterations 24000 to 25000 for a given relaxation-free test. The time step is chosen to be lower than $1 /(10 f)$ where $f=\max \left(\left|\omega_{12}\right|, \gamma^{\text {coll }}\right)$.
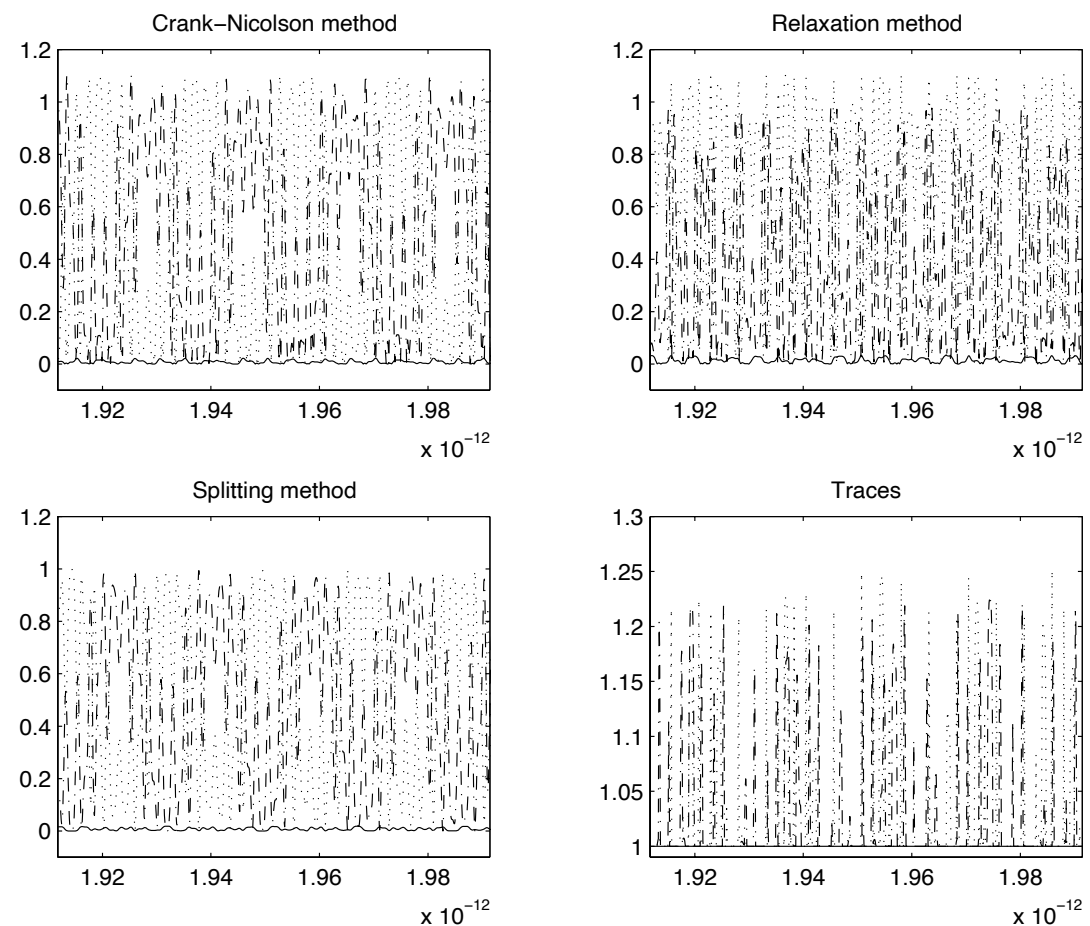

FIG. 1. Comparison of different numerical schemes The three first plots represent the time evolution of populations for three-level atoms and for different numerical schemes. The last plot represents the time evolution of the trace for the Crank-Nicolson scheme (dash-point), the relaxation scheme (points) and the splitting scheme (solid).

The details of the evolution is not important for our demonstration. Solutions are highly oscillatory due to the fact that the field is monochromatic and its frequency is exactly matching the matter transitions. The important point is the range of the values for the populations. We see that the Crank-Nicolson method and the relaxation method do not preserve positiveness (i.e. populations do not lie in the interval $[0,1])$ as well as the trace property while the splitting method does. Estimates on coherences are not presented here but are wrong too. Besides CPU times are 23 seconds for the Crank-Nicolson scheme, 27 seconds for the relaxation scheme (and indeed no improvement was expected with a given field) and 11 seconds for the splitting scheme. Last the dynamics seems to be the same (up to a small shift) for the Crank-Nicolson method and the splitting method but qualitatively different 
for the relaxation scheme. For all these reasons the splitting method is used for the next tests.

Simulations with different relaxation rates. This paragraph is devoted to the demonstration that being able to handle different relaxation rates helps to describe the wide variety of transient behavior of systems. In figures 2 and 3 is represented the transient and long time evolution of a medium (where initially $\rho_{11}=1$ ) under the influence of a wave paquet with leading frequency $5 \omega_{31}$. Dipole matrix elements are $p_{12}=0, p_{13}=10^{-28}$ and $p_{23}=10^{-29}$. In both simulations $W_{13}=0$ and $\gamma^{\text {coll }}=0$.
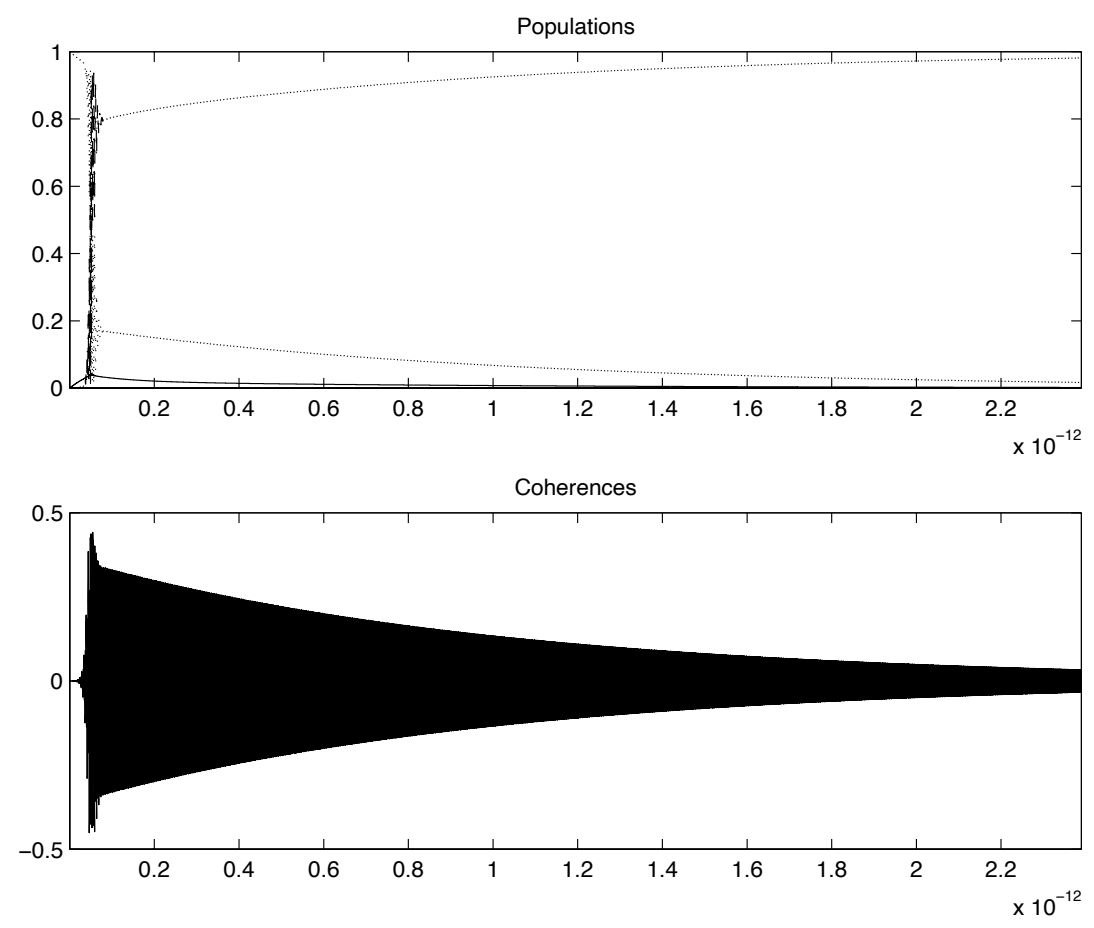

FIG. 2. Different relaxation rates. Relaxation rates are $W_{21}=10^{12}$ and $W_{32}=10^{13}$.

The long time behavior is of course also slightly affected by the change of the matrix $W$ according to equation (5).

\section{CONCLUSION}

The introduction of more than two levels in a Bloch model induces some new modeling problems. Multi-level codes are however necessary to model physical phenomena as, for example, coherence transfer [2] or Raman effects. Numerical models that were used up to now for two-level atoms are not able to preserve natural properties for three or more level atoms, the main (or more visible) of which being that populations may not lie between 0 and 1 . We found out an alternative method that has not only the advantage of preserving positiveness properties but also allows some gain in computational time. In the case of a coupling with Maxwell equations it also allows to decouple the computation of the field and the density matrix thus leading to substantial gains in computational time but also to the efficient 

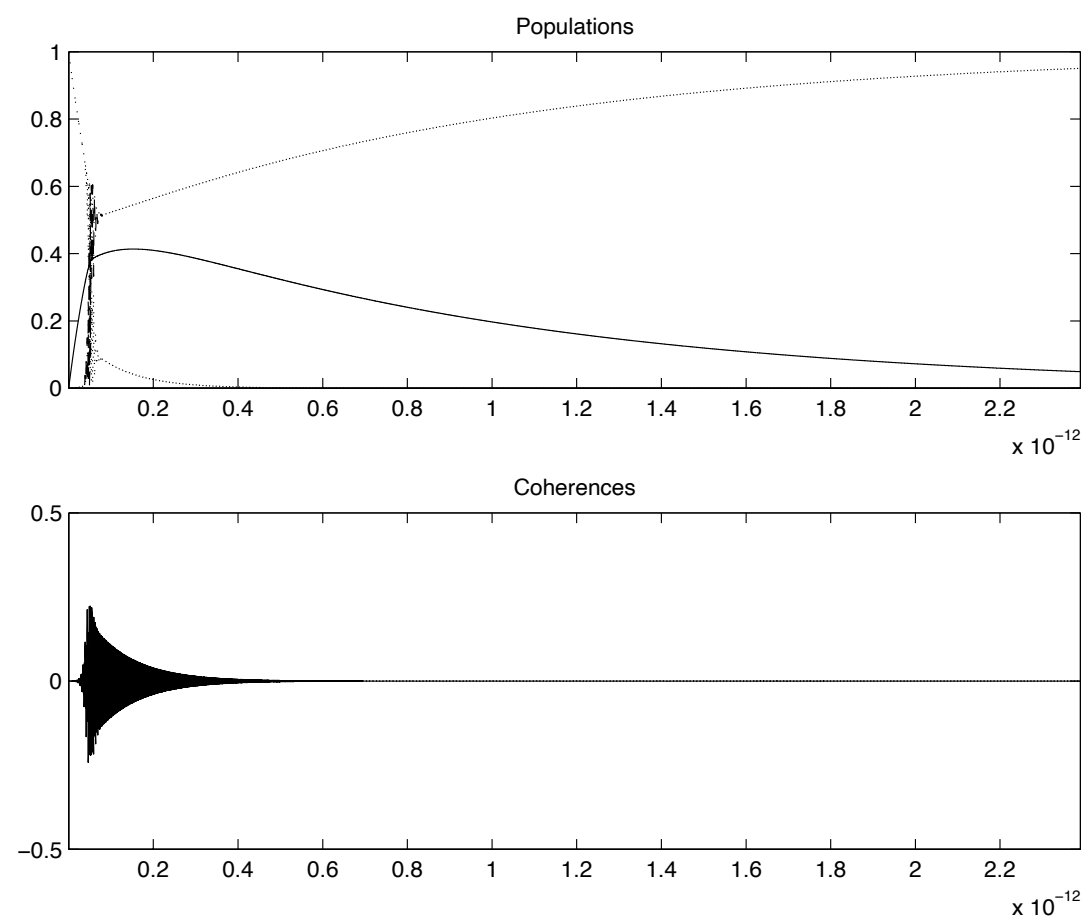

FIG. 3. Different relaxation rates. Relaxation rates are $W_{21}=10^{13}$ and $W_{32}=10^{12}$.

parallelization of the code, which could be useful for one-dimensional codes but even more for two or three-dimensional codes.

\section{APPENDIX: FADEEV FORMULAE FOR TWO, THREE AND FOUR LEVEL ATOMS}

We use Faddev formula to compute matrix $M_{N}=\left(I-\frac{i \delta t}{2 \hbar} V\right)\left(I+\frac{i \delta t}{2 \hbar} V\right)^{-1}$ for $N$ level atoms. This formula gives an algorithm to compute matrix inverses. Indeed, given a matrix $A, A^{-1}=\frac{1}{p_{N}} B_{N-1}$ where

$$
\begin{array}{ccc}
A_{1}=A, & p_{1}=\operatorname{Tr} A_{1}, & B_{1}=A_{1}-p_{1} I, \\
A_{2}=A B_{1}, & p_{2}=\frac{1}{2} \operatorname{Tr} A_{2}, & B_{2}=A_{2}-p_{2} I, \\
\vdots & \vdots & \vdots \\
A_{N}=A B_{N_{1}}, & p_{N}=\frac{1}{N} \operatorname{Tr} A_{N}, & B_{N}=A_{N}-p_{N} I=0 .
\end{array}
$$

In our case $A=I+\varepsilon p$ and $\varepsilon=\frac{i \delta t}{2 \hbar} E$ ) (in the case of multidimensional spaces, contributions in each direction should be added). Some simplifications are due to the fact that $\operatorname{Tr} p=0$. Computations lead to

$$
\begin{aligned}
& M_{2}=I+\frac{\varepsilon^{2} \operatorname{Tr} p^{2} I-2 \varepsilon p}{1-\frac{1}{2} \varepsilon^{2} \operatorname{Tr} p^{2}}, \\
& M_{3}=I+\frac{-\frac{2}{3} \varepsilon^{3} \operatorname{Tr} p^{3} I-2 \varepsilon p+2 \varepsilon^{2} p^{2}}{1-\frac{1}{2} \varepsilon^{2} \operatorname{Tr} p^{2}+\frac{1}{3} \varepsilon^{3} \operatorname{Tr} p^{3}},
\end{aligned}
$$




$$
\begin{aligned}
M_{4}=I+\frac{\left(-\frac{2}{3} \varepsilon^{3} \operatorname{Tr} p^{3}-\frac{1}{4} \varepsilon^{4}\left(\operatorname{Tr} p^{2}\right)^{2}+\frac{1}{2} \varepsilon^{4} \operatorname{Tr} p^{4}\right) I}{1-\frac{1}{2} \varepsilon^{2} \operatorname{Tr} p^{2}+\frac{1}{3} \varepsilon^{3} \operatorname{Tr} p^{3}+\frac{1}{8} \varepsilon^{4}\left(\operatorname{Tr} p^{2}\right)^{2}-\frac{1}{4} \varepsilon^{4} \operatorname{Tr} p^{4}} \\
-\frac{\left(2-\varepsilon^{2} \operatorname{Tr} p^{2}\right) \varepsilon p+2 \varepsilon^{2} p^{2}-2 \varepsilon^{3} p^{3}}{1-\frac{1}{2} \varepsilon^{2} \operatorname{Tr} p^{2}+\frac{1}{3} \varepsilon^{3} \operatorname{Tr} p^{3}+\frac{1}{8} \varepsilon^{4}\left(\operatorname{Tr} p^{2}\right)^{2}-\frac{1}{4} \varepsilon^{4} \operatorname{Tr} p^{4}} .
\end{aligned}
$$

\section{REFERENCES}

1. Bidégaray, B. Numerical time coupling of Maxwell and Bloch equations, in preparation.

2. Bidégaray, B., Bourgeade, A., Reignier, D. and Ziolkowski R. (2000). Multilevel Maxwell-Bloch simulations, accepted by the "Fifth International Conference on Mathematical and Numerical Aspects of Wave Propagation", Santiago de Compostela, July 2000.

3. Bloembergen, N. (1996). Nonlinear Optics, World Scientific.

4. Boyd, R.W. (1992). Nonlinear Optics, Academic Press.

5. Guo, B.-Y., Martín, I., Pérez-García, and Vázquez L. (1996). Numerical Solution of the Maxwell-Bloch Laser Equation, J. Comput. Phys., 129, pp. 181-189.

6. Hioe, F.T. and Eberly, J.H. (1981). N-level coherence vector and higher conservation laws in quantum optics and quantum mechanics, Phys. Rev. Lett., 47, pp. 838-841

7. Loudon, R. (1973). The Quantum Theory of Light, Oxford Science Publications.

8. Martín, I., Pérez-García, V.M., Guerra, J.M., Tirado, F. and Vázquez L. (1995). Numerical simulations of the Maxwell-Bloch laser equations, in "Fluctuations phenomena : Disorder and nonlinearity", Eds. L. Vázquez and A. Bishop, Nonlinear Science Series, World Scientific.

9. Nagra, A.S. and York, R.A. (1998). FDTD Analysis of Wave Popagation in Nonlinear Absorbing and Gain Media, IEEE Trans. Antennas Propagat., 46, pp. 334-340.

10. Ziolkowski, R.W., Arnold, J.M. and Gogny, D.M. (1995). Ultrafast pulse interaction with two-level atoms, Phys. Rev. A, 52, pp. 3082-3094. 